УДК 621.039

В. И. Скалозубов ${ }^{1}$, В. А. Тарасов ${ }^{2}$,

С. И. Косенко ${ }^{2}$, С. А. Чернеженко ${ }^{2}$,

А. А. Какаев ${ }^{2}$, В. Ю. Кочнева ${ }^{1}$

${ }^{1}$ Институт проблем безопасности АЭС НАН Украины, г. Киев, Украина

2 Одесский национальный политехнический университет, г. Одесса, Украина

Методические основы адаптации MOX-топлива в аварийных режимах для реакторов типа ВВЭР

Предложен критериальный метод адаптации «непроектного» для ВВЭР МОХ-топлива в аварийных режимах (в том числе при тяжелых авариях с повреждением ядерного топлива) на основе детерминистических оценок условий устойчивого расхождения температурных зависимостей MOX- и уранового топлива в процессе развития аварии. Применение метода позволит определить перечень аварий (этапов аварий) с МОХ-топливом, требующих дополнительного детализированного моделирования, а также допустимое обогащение плутонием МOХ-топлива для энергоблоков АЭС с ВВЭР.

Ключевые слова: адаптация, авария, ядерное MOX-топливо, урановое ядерное топливо, водо-водяной энергетический реактор (ВВЭР).

В. І. Скалозубов, В. О. Тарасов, С. І. Косенко, С. А. Чернеженко, А. О. Какаєв, В. Ю. Кочнєва

\section{Методичні основи адаптації МОХ-палива в аварійних режимах для реакторів типу ВBEP}

Запропоновано критеріальний метод адаптації «непроектного» для BBEP MOX-палива в аварійних режимах (зокрема в разі важких аварій з пошкодженням ядерного палива) на основі детерміністичних оцінок умов стійкого розходження температурних залежностей МОХ та уранового палива в процесі розвитку аварії. Застосування методу дасть змогу визначити перелік аварій (етапів аварій) з МOХ-паливом, що потребують додаткового деталізованого моделювання, а також допустиме збагачення плутонієм MOX-палива для енергоблоків AEC з $B B E P$.

Ключов і слов в: адаптація, аварія, ядерне MOX-паливо, уранове ядерне паливо, водо-водяний енергетичний реактор (ВВEP).

() В. И. Скалозубов, В. А. Тарасов, С. И. Косенко, С. А. Чернеженко, А. А. Какаев, В. Ю. Кочнева, 2015
B настоящее время в Украине на четырех АЭС эксплуатируется 15 энергоблоков с ядерными реакторами типа ВВЭР, использующих по проекту оксидно-урановое топливо. В связи со сложившимися в последнее время обстоятельствами в ближайшей перспективе возможен частичный или полный переход на «непроектное» ядерное топливо на украинских АЭС, так как запасы проектного российского ядерного топлива истекают к концу 2015 года. Одним из вариантов замены проектного ядерного топлива рассматривается и МOX-топливо, что требует дополнительного глубокого изучения и научно-технического обоснования с учетом особенностей эксплуатации плутониевого топлива на основе соответствующего анализа безопасности и непосредственной поэтапной адаптации на натурных объектах.

Одно из ограничений обоснованной адаптации МОХ-топлива для украинских АЭС связано с необходимостью экстраполяции результатов адаптации в рабочих режимах реактора на условия аварийных режимов: имеющиеся различия нейтронно-физических характеристик уранового и плутониевого топлива могут определить различия как непосредственно протекания аварийных процессов, так и соответствующих мероприятий по управлению авариями с учетом конструкционно-технических особенностей ВВЭР (например, [1-11]), что в конечном итоге определяет актуальность разработки методов адаптации «непроектного» МОХ-топлива в аварийных режимах.

Уроки Фукусимской аварии в марте 2011 года также определили актуальность вопросов адаптации MOX-топлива: наиболее мощные разрушительные взрывы по официальной информации эксплуатирующей организации ТЕРСО [2] произошли именно на объектах, частично загруженных МOX-топливом.

Дополнительные технические обоснования адаптации «непроектного» ядерного топлива требуют, в общем случае, проведения в полном объеме моделирования и анализа всех аварийных режимов (включая возможные изменения химического состава и обогащения топлива в процессе эксплуатации). Поэтому определенный интерес представляют вопросы предварительного оперативного определения условий и аварийных режимов, в которых влияние эффектов «непроектного» ядерного топлива может быть наиболее существенным, что, возможно, сократит объемы детализированного анализа безопасности.

В данной работе представлены методические основы адаптации МOX-топлива в аварийных режимах для реакторных установок с ВВЭР.

Основные положения предлагаемого метода адаптации «непроектного» для ВВЭР МОХ-топлива в аварийных режимах заключаются в следующем.

1. Различие нейтронно-физических свойств МОХтоплива и проектного топлива определяет различие в общем случае температурных зависимостей мощности внутренних энерговыделений ядерных реакций и условий межфазного тепломассообмена в процессе аварий как с «плотным» реакторным контуром (отказы аварийной защиты реактора, полное обесточивание энергоблока, разрывы паропроводов второго контура и т. п.), так и с «неплотным» реакторным контуром (течи реактора и оборудования или трубопроводов реакторного контура, межконтурные течи и пр.).

2. Критерием адаптации «непроектного» МOX-топлива в аварийных процессах определяются условия отсутствия устойчивого расхождения текущих температурных зависимостей по отношению к «проектному» урановому топливу. 
3. Тепловыделяющий элемент (твэл) консервативно моделируется как сосредоточенная система с максимальной температурой ядерного топлива $T_{\text {я.т }}$, а также принимаются консервативные значения теплофизических свойств ядерного топлива.

Расчетная модель критерия адаптации приведена на рис. 1.

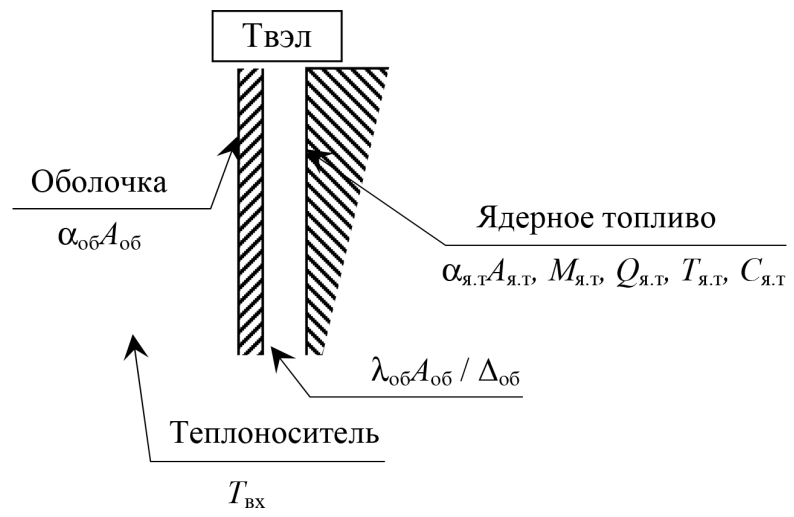

Рис. 1. Расчетная модель критерия адаптации

Уравнение сохранения тепловой энергии в формате изменения максимальной (по высоте твэла) температуры ядерного топлива $T_{\text {я.т }}$

$$
M_{\text {я.T }} C_{\text {я.Т }}\left(T_{\text {я.Т }}\right) \frac{\mathrm{d} T_{\text {я.Т }}}{\mathrm{d} t}=Q_{\text {я.Т }} M_{\text {я.Т }}\left(T_{\text {я.Т }}\right)-\alpha_{0}\left(T_{\text {я.Т }}\right)\left[T_{\text {я.Т }}-T_{\text {вх }}\right]
$$

при начальных условиях

$$
T_{\text {я.Т }}(t=0)=T_{\text {я.Т } 0},
$$

где $M_{\text {я.Т }}, C_{\text {я.т }}, T_{\text {я.Т }}, Q_{\text {я.т }}-$ масса, удельная теплоемкость, температура и удельная мощность внутренних энерговыделений ядерного топлива соответственно; $T_{\text {вх }}-$ температура теплоносителя на входе в активную зону ядерного реактора; $\alpha_{0}-$ приведенный общий коэффициент теплопередачи между ядерным топливом и теплоносителем:

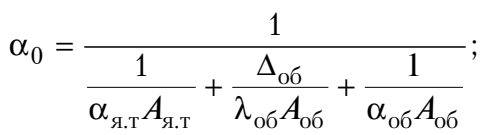

$\alpha_{\text {об}}, \alpha_{\text {я.т }}-$ коэффициенты теплоотдачи от оболочки и ядерного топлива соответственно; $\lambda_{\text {об }}, \Delta_{\text {об }}-$ теплопроводность и толщина оболочки твэла; $A_{\text {об }}, A_{\text {я.т }}-$ площадь поверхности оболочки твэла и ядерного топлива соответственно; $t-$ время развития аварийного режима;

В критериальном формате уравнения (1) и (2) для аварийного процесса длительностью $t_{A}$ имеют вид

$$
\begin{gathered}
\frac{d \mathbf{T}_{\text {я.Т }}}{d \mathbf{t}}=\mathbf{K}_{N}-\mathbf{K}_{T}\left[\mathbf{T}_{\text {я.Т }}-\mathbf{T}_{\mathrm{BX}}\right], \\
\mathbf{T}_{\text {я.Т }}(\mathbf{t}=0)=\mathbf{T}_{\text {я.Т } 0},
\end{gathered}
$$

где $\mathbf{T}_{\text {я.т }}=T_{\text {я.т } 0} / T_{m} ; \mathbf{t}=t / t_{A} ; T_{m}=\frac{Q_{m}\left(\mathrm{U}_{2} \mathrm{O}\right) t_{A}}{C_{\text {я.т }}(\mathrm{MOX})} ; \mathbf{K}_{N}=\frac{Q_{\text {я.т }}(\mathrm{MOX})}{Q_{m}\left(\mathrm{U}_{2} \mathrm{O}\right)} ;$ $\mathbf{K}_{T}=\frac{\alpha_{0} t_{A}}{C_{\text {я.Т }} M_{\text {я.Т }}(\mathrm{MOX})} ; Q_{m}\left(\mathrm{U}_{2}\right)-$ масштаб мощности внутренних энерговыделений $\mathrm{U}_{2}$-топлива.
Условия устойчивого расхождения текущих значений температур MOX- и $\mathrm{U}_{2}$-топлива можно оценить из общих положений теории неустойчивости в «малом» приближении [12]: если флуктуационное возмущение температуры ядерного топлива $\left(\delta T_{\text {я.т }}<<T_{\text {я.т }}\right)$ приводит к устойчивому росту относительных текуших значений температур МOXи $\mathrm{U}_{2}$-топлива в процессе развития аварийных процессов, то критерий адаптации не выполняется.

В формате флуктуационных возмущений уравнение (3) имеет вид

$$
\frac{d \delta \mathbf{T}_{\text {я.Т }}}{d \mathbf{t}}=\frac{d \mathbf{K}_{N}}{d \mathbf{T}_{\text {я.Т }}} \delta \mathbf{T}_{\text {я.Т }}-\mathbf{K}_{T} \delta \mathbf{T}_{\text {я.Т }} .
$$

Решение (5) имеет вид

$$
\delta \mathbf{T}_{\text {я.Т }} \approx \exp \left[\left(\frac{d \mathbf{K}_{N}}{d \mathbf{T}_{\text {я.Т }}}-\mathbf{K}_{T}\right) \mathbf{t}\right] .
$$

Из решения (6) следует критерий адаптации

$$
\frac{d \mathbf{K}_{N}}{d \mathbf{T}_{\text {g.T }}} \leq \mathbf{K}_{T} .
$$

Определяющим параметром критерия адаптации (7) при подобии «внешних» условий тепломассообмена является отношение температурных зависимостей мощности внутренних энерговыделений $\mathrm{MOX}$ - и $\mathrm{U}_{2}$-топлива. Мощность внутренних энерговыделений для реакторного топлива в зависимости от температуры ядерного топлива в предположении пространственной однородности нейтронного поля в активной зоне реактора и при пренебрежении уменьшением концентрации ядер, вызванным термическим расширением, можно оценить с помощью следующего выражения [1]:

$$
Q_{\text {я.Т }}\left(T_{\text {я.Т }}\right)=\Phi \sum_{i} Q i\left\langle\sigma_{f}^{i}\right\rangle N^{i}
$$

где $\Phi-$ приведенная по размерности плотность потока нейтронов; $Q_{i}-$ усредненная тепловая энергия деления одного ядра $i$-нуклида реакторного топлива; $\left\langle\sigma_{f}^{i}\right\rangle-$ усредненное по энергетическому спектру нейтронов сечение деления $i$-нуклида; $N^{i}-$ концентрация ядер $i$-нуклида.

Предварительные расчеты показали, что определяющим параметром расхождения относительной мощности внутренних энерговыделений для плутониевого и уранового топлива является существенное различие в определенном диапазоне температур значений сечения деления нуклидов, что соответствует известным результатам [7-9].

\section{Выводы}

Предложенный метод адаптации позволяет при варьировании значений температуры ядерного топлива (включая высокие значения, соответствующие разрушению ядерного топлива, - тяжелые аварии), а также уровня обогащения плутонием ядерного топлива оперативно оценить перечень аварий (этапов аварийных процессов) c MOX-топливом, требующих дополнительного детализированного моделирования, и допустимое обогащение MOX-топлива плутонием для энергоблоков АЭС с ВВЭР. 
Кроме того, предложенный метод позволяет оперативно оценить влияние на аварийные режимы изменения химического состава и обогащения проектного ядерного топлива в процессе эксплуатации.

В последующих работах будут приведены соответствующие результаты совершенствования предложенного метода и расчетного моделирования.

\section{Список использованной литературы}

1. Различие температурных зависимостей плотностей теплового источника мокс-топлива и диоксидного топлива и связанные с этим особенности аварии на третьем блоке АЭС «Фукусима-1» / В. Д. Русов, В. А. Тарасов, С. А. Чернеженко, А. А. Какаев, Е. В. Гречан, С. И. Косенко, О. И. Пантак // Мат-лы 3-й междунар. науч.-практ. конф. «Повышение безопасности и эффективности атомной энергетики», 24-28 сент. 2012 г., Одесса, Украина. Чернобыль : ИПБ АЭС НАНУ, 2013.

2. Анализ причин и последствий аварии на АЭС Fukushima как фактор предотвращения тяжелых аварий в корпусных реакторах / В. И. Скалозубов, А. А. Ключников, В. Н. Ващенко, С. С. Яровой. - Чернобыль : ИПБ АЭС НАНУ, 2012. -280 с.

3. Вейнберг А. Физическая теория ядерных реакторов : пер. с англ. / А. Вейнберг, Е. Вигнер. - М. : Изд-во иностр. лит-ры, 1961. $-733 \mathrm{c}$

4. Основы теории и методы расчета ядерных энергетических реакторов / Г. Г. Бартоломей, Г. А. Бать, В. Д. Байбаков, М. С. Алхутов. - М. : Энергоатомиздат, 1989. - 512 с.

5. Фейнберг С. М. Теория ядерных реакторов / С. М. Фейнберг, С. Б. Шихов, В. Б. Троянский. - М. : Атомиздат, 1978. - 400 с.

6. Групповые константы для расчета реакторов и защиты / П. Л. Абагян, Н. О. Базазянц, М. Н. Николаев, А. М. Цибуля. М. : Энергоиздат, 1981. - 139 с.

7. Русов В. Д. Режимы с обострением в уран-плутониевой делящейся среде технических ядерных реакторов и геореактора / В. Д. Русов, В. А. Тарасов, С. А. Чернеженко // Вопросы атомной науки техники. - 2011. - № 2(97). - С. 112-121. - (Серия «Физика радиационных повреждений и радиационное материаловедение»).

8. The Temperature Dependences Distinction of Thermal Source Densities of MOX-Fuel and Dioxide-Fuel and Related with It the Features of NPP «Fukusima-1» Third Unit Accident / V. D. Rusov, V. A. Tarasov, S. A. Chernezhenko, A. A. Kakaev, E. V. Grechan, S. I. Kosenko, O. I. Pantak // Proc. of $4^{\text {th }}$ Int. Conf. «Current Problems in Nuclear Physics and Atomic Energy» (NPAE-Kyiv2012), 10-14 September 2012, Kyiv, Ukraine. - Kyiv : Institute for Nuclear Research, 2013. - P. 479-483.

9. Fukushima Plutonium Effect and Blow-Up Regimes in NeutronMultiplying Media / V. D. Rusov, V. A. Tarasov, V. M. Vaschenko, E. P. Linnik, T. N. Zelentsova, M. E. Beglaryan, S. A. Chernegenko, S. I. Kosenko, P. A. Molchinikolov, V. P. Smolyar, E. V. Grechan // World Journal of Nuclear Science and Technology. - 2013. - № 3. P. 9-18. - arXiv:1209.0648v1 [nucl-th].

10. Украинцев В. Ф. Эффекты реактивности в энергетических реакторах / В. Ф. Украинцев. - Обнинск : ИАТЭ, 2000. - 60 с.

11. Кесслер Г. Ядерная энергетика : пер. с англ. - М. : Энергоатомиздат, 1986. - 264 с.

12. Скалозубов В. И. Основы управления запроектными авариями с потерей теплоносителя на АЭС с ВВЭР / В. И. Скалозубов, А. А. Ключников, В. Н. Колыханов. - Чернобыль : ИПБ АЭС НАНУ, 2010. - $400 \mathrm{c}$.

\section{References}

1. Rusov, V.D., Tarasov, V.A., Chernezhenko, S.A., Kakaev, A.A., Grechan, E.V., Kosenko, S.I., Pantak, O.I. (2013), "Distinction of Temperature Dependences for Thermal Source Densities of MOX Fuel and Dioxide Fuel and Related Features of Accidents at Fukushima-1 Unit 3" [Razlichiie temperaturnykh zavisimostei plotnostei teplovogo istochnika moks-topliva $\mathrm{i}$ dioksidnogo topliva $\mathrm{i}$ sviazannyie $\mathrm{s}$ etim osobennosti avarii na tretiem bloke AES Fukusima-1], Proceedings of the $3^{\text {rd }}$ International Scientific and Practical Conference "Nuclear Power Engineering Safety and Efficiency Improvement" (Odessa, Ukraine, 24-28 September, 2012), Chornobyl: Institut problem bezpeky AES NAN Ukrainy (Institute for Safety Problems of Nuclear Power Plants). (Rus)

2. Skalozubov, V.I., Klyuchnikov, A.A., Vaschenko, V.N., Yarovoi, S.S. (2012), "Analysis of Causes and Consequences of Fukushima Accident for Prevention of Severe Accidents in Vessel-Type Reactors" [Analiz prichin i posledstvii avarii na AES Fukushima kak faktor predotvrascheniya tiazhelykh avarii v korpusnykh reaktorakh], Institute for Safety Issues of Nuclear Power Plants, Chornobyl, $280 \mathrm{p}$.

3. Weinberg, A.M., Wigner, E.P. (1961), "The Physical Theory of Neutron Chain Reactors", Izdatel'stvo inostrannoi literatury, Moscow, 733 p. (Rus)

4. Bartolomei, G.G., Bat, G.A., Baibakov, V.D., Alhutov, M.S. (1989), Theory and Design Methods of Nuclear Reactors [Osnovy teorii i metody rascheta yadernykh energeticheskikh reaktorov], Energoatomizdat, Moscow, 512 p. (Rus)

5. Feinberg, S.M., Shihov, S.B., Troyanskii, V.B. (1978), Theory of Nuclear Reactors [Teoriia yadernykh reaktorov], Atomizdat, Moscow, 400 p. (Rus)

6. Abaghian, P.L., Bazazyanc, N.O., Nikolaev, M.N., Tsibulya, A.M. (1981), Group Constants for Reactors and Shielding Calculation [Gruppovyie konstanty dlia rascheta reaktorov i zaschity], Energoizdat, Moscow, 139 p. (Rus)

7. Rusov, V.D., Tarasov, V.A., Chernezhenko, S.A. (2011), "Sharpening Modes in Uranium-Plutonium Fissile Medium of Engineering Nuclear Reactors and Georeactor" [Rezhimy s obostreniiem v uran-plutoniievoi deliascheisia srede tekhnicheskikh yadernykh reaktorov i georeaktora,], Voprosy atomnoi nauki i tekhniki, No. 2(97), pp. 112-121. (Rus)

8. Rusov, V.D., Tarasov, V.A., Chernezhenko, S.A., Kakaev, A.A., Grechan, E.V., Kosenko, S.I., Pantak, O.I. (2013), Temperature Dependences Distinction of Thermal Source Densities of MOX-Fuel and Dioxide-Fuel and Related Features of Accident at NPP "Fukushima-1" Unit 3, Proceedings of the $4^{\text {th }}$ International Conference "Current Problems in Nuclear Physics and Atomic Energy" (NPAE-Kyiv2012), (Kyiv, Ukraine, 10-14 September, 2012,), Institute for Nuclear Research, Kyiv, pp. 479-483.

9. Rusov, V.D, Tarasov, V.A., Vaschenko, V.M., Linnik, E.P., Zelentsova, T.M., Beglaryan, M.E., Chernegenko, S.A., Kosenko, S.I., Molchinikolov, P.A., Smolyar, V.P., Grechan, E.V. (2013), "Fukushima Plutonium Effect and Blow-Up Regimes in Neutron-Multiplying Media", World Journal of Nuclear Science and Technology, No.3, pp. 9-18, arXiv:1209.0648v1 [nucl-th].

10. Ukraintsev, V.F. (2000), Reactivity Effects in Power Reactors [Effekty reaktivnosti v energeticheskikh reaktorakh], Obninsk Institute for Nuclear Power Engineering, Obninsk, 60 p. (Rus)

11. Kessler, G. (1986), Nuclear Energy [Russian translation], Energoatomizdat, Moscow, 264 p.

12. Skalozubov, V.I., Klyuchnikov, A.A., Kolykhanov, V.N. (2010), "Management of Beyond Design-Basis Accidents with Loss of Coolant at NPPs with WWER" [Osnovy upravleniya zaproektnymi avariyami s poterei teplonositelya na AES s VVER], Institute for Safety Issues of Nuclear Power Plants, Chornobyl, 400 p. (Rus)

Получено 24.10.2014. 